\title{
First-Principles Calculation of Contact Electrification and Validation by Experiment
}

Xiaozhou Shen, Andrew E. Wang, R. Mohan Sankaran, Daniel J. Lacks

Department of Chemical and Biomolecular Engineering, Case Western Reserve University,

Cleveland OH 44106, United States

E-mail: daniel.lacks@case.edu and mohan@case.edu

Contact electrification is one of the most well-known phenomena in physics and examples arise in almost every industry. However, the scientific basis of contact charging is unknown. Here, we present a theoretical study of contact electrification, supported by experiments, to calculate for the first time charge transfer between material surfaces from first principles physics. Electronic structure calculations and experiments are performed on single-crystal alumina (sapphire) and silicon oxide (quartz) surfaces, which have well-ordered strutures that enable rigorous modeling. Both experiments and calculations show that sapphire charges positively and quartz charges negatively. The calculations cannot determine the magnitude of charge densities remaining on separated surfaces from first principles, as these are non-equilibrium effects, but our analysis suggests that the calculation results are consistent with the experimental charge densities of 10 $\mu \mathrm{C} / \mathrm{m}^{2}$. These results indicate the possibility of quantitatively predicting and explaining contact electrification from only the molecular structure of material surfaces.

KEYWORDS: Contact Electrification; Sapphire; Quartz; Electronic structure calculations. 


\section{Introduction}

When two initially uncharged material surfaces come in contact and then separate, an exchange of charges can occur such that one surface becomes positively charged and the other becomes negatively charged. This basic description of "contact electrification” is one of the most well-known phenomena in physics, and examples arise in almost every industry. However, the scientific basis of contact charging remains unknown. ${ }^{1}$ It is not clear whether the species transferred between surfaces that lead to charging are electrons, ions, or bits of material, ${ }^{2,3}$ how the direction of net charge transfer depends on material properties, ${ }^{4}$ or why the charge is heterogeneous both in terms of polarity and magnitude across the surface. ${ }^{5}$ It is possible that various mechanisms may occur, with different species dominating in different situations. For example, Baytekin et al. ${ }^{6}$ recently showed that when polytetrafluoroethylene (PTFE) beads are rolled in a polystyrene dish, the beads charge negatively after a short amount of rolling, but then become positively charged after a longer amount of rolling. The initial charging may be due to electrons or ions transferring; however, the shift in the polarity of the charging at later times was shown to be due to nanoscale patches of material being torn off and transferred to the other surface. Thus, even in a single experiment, more than one mechanism can contribute, complicating the charging process.

To fully understand these issues, it will be necessary to model contact charging with a rigorous approach that draws only on the most fundamental laws of physics, with no empiricism or experimental input. Experiments alone cannot resolve these issues. Ideally, the modeling approach should take as input only the molecular description of the materials involved, and determine the contact charging behavior from this information alone. Since electron transfer can play a role, the approach must explicitly model the electron states of the system, which requires 
quantum mechanical methods. And since electron states in macroscopic solids are delocalized, the modeling must consider a system large enough to behave like a macroscopic solid, rather than just a molecular fragment. Such quantum mechanical calculations on extended systems are extremely computationally intensive, and to become at all feasible we must consider the simplest systems possible. Unfortunately, most previous experiments on contact charging addressed polymers, which are very complex because they are structurally heterogeneous, with a distribution of molecular weights and branching; often have semi-crystalline structures, which are more complicated than either fully crystalline or fully amorphous structures; are in nonequilibrium states, such that the structures change with time and are sample-history-dependent; and contain impurities that arise either during processing, such as residuals of catalyst and catalyst support, or from chemical degradation due to oxidation.

Here, we present contact charging results for well-defined materials that can be addressed with both rigorous first-principles calculations and experiments. We examine contact between particular crystallographic surfaces of single-crystal quartz $\left(\mathrm{SiO}_{2}\right)$ and single-crystal sapphire $\left(\mathrm{Al}_{2} \mathrm{O}_{3}\right.$; we use the term 'sapphire' to describe the crystal structure, which is also known as corundum and $\alpha-\mathrm{Al}_{2} \mathrm{O}_{3}$ ). Our calculations and experiments both find sapphire charging positively and quartz charging negatively. To our knowledge, this is the first corroboration of contact charging between rigorous first-principles calculations and experiments. While this study focuses on well-defined materials that facilitate modeling, our approach may eventually find application to other materials including polymers. 


\section{Methods}

\section{Calculations}

We addressed contact charging using a first-principles approach that takes as input only the molecular description of the component materials involved. We modeled the complete threedimensional structure of the solid system at the electronic structure level. The electron states were determined, and from the electron states the energy of the system and the forces on the atoms were obtained. Quantum mechanical methods are required to determine the electron states; i.e., the many-body Schrodinger's equation is solved within a set of approximations. The approximations used were based on density functional theory and the use of an atomic orbital basis set. In particular, the Kohn-Sham equations were solved with the PBE functional ${ }^{7}$ and the double-zeta with polarization functions basis set. The calculations were carried out with the SIESTA software. ${ }^{8}$

The system we model is shown in Figure 1. The interface consists of the (0001) crystallographic planes of quartz and sapphire oriented parallel to each other. Periodic boundary conditions are used, and so the surfaces are infinitely wide. It is fortuitous that we can model this system, and we are only able to do so because quartz and sapphire both have monoclinic structures with similar $a$ lattice parameters: $a=4.91 \AA$ for quartz ${ }^{9}$ and $a=4.79 \AA$ for sapphire. ${ }^{10}$ Our simulation cell extended two unit cells in the directions parallel to the surface; periodic boundary conditions were applied in these directions with the repeat unit $9.516 \AA$. In the direction perpendicular to the surfaces, the materials were finite in thickness, with the layers of quartz and sapphire each being two unit cells thick. The surfaces were hydroxylated, and we began our simulations with structures previously reported for quartz ${ }^{11}$ and sapphire ${ }^{12}$ at ambient conditions. The distance between the quartz and sapphire surfaces was varied, and ranged from 
$1.4 \AA$ to $10 \AA$. Periodic boundary conditions were also used in the direction perpendicular to the surfaces (these boundary conditions must be applied in all dimensions); however, the repeat distance was chosen to be large enough $\left(50 \AA^{3}\right)$ to limit interactions to only one quartz-sapphire interface (and not with the periodic image). The simulation cell included 268 atoms, with 132 atoms in the quartz slab and 136 atoms in the sapphire slab.

We first obtained the minimum energy structures for the separate quartz and sapphire slab systems; the energy-minimized structures agreed well with previous work for quartz ${ }^{11}$ and sapphire $^{12}$. In our calculations of the complete system with both quartz and sapphire slabs, we kept the atom positions fixed at these minimum energy positions.

\section{Experiments}

Contact charging experiments were carried out in a humidity-controlled acrylic glove box (Electro-Tech Systems Inc.) with feedthroughs for electrical connections and gas fittings. The humidity inside the glove box was controlled with an air stream of controlled humidity. The lowest humidity was obtained by flowing only dry air. To create higher humidity, a wet air inlet stream was used. The wet air stream was created by bubbling dry air through a heated water bath to reach a desired saturated water vapor concentration. A fan inside the glove box was used to enhance mixing of the inlet air stream with the background air in the glove box. Once the desired steady-state humidity was established in the glove box, the air flow was shut off and experiments were performed in a static (no flow) condition. The humidity and temperature in the glove box were monitored with a digital probe (EQ-RH-606B). This method was able to achieve desired set points between $5 \%$ to $95 \% \pm 0.3 \%$. 
We focused on single-crystal oxides for this study. The samples were $1 \mathrm{~cm}^{2}, 0.5 \mathrm{~mm}$ thick, single crystallographic cuts of sapphire [0001] and quartz [0001] (MTI Corporation), with one side polished; some experiments were also carried out with MgO periclase (001) (MTI Corporation). Samples were cleaned before contact by the RCA ${ }^{13,14}$ wafer cleaning recipe, omitting the oxide strip setup. Briefly, the samples were immersed in a 1:1:5 (v/v/v) mixture of 29\% ammonia, 30\% hydrogen peroxide, and distilled water and sonicated at $25{ }^{\circ} \mathrm{C}$ for 20 min. The samples were then rinsed and submerged in distilled water for $1 \mathrm{~min}$. They were then treated in a 1:1:6 (v/v/v) mixture of $15 \mathrm{M}$ hydrochloric acid, 20\% hydrogen peroxide by sonication at 25 ${ }^{\circ} \mathrm{C}$ for $20 \mathrm{~min}$. The samples were then rinsed and submerged in distilled water for 1 min. Finally, the samples were removed and any residual water was blown off by compressed dry air for 5 seconds on each face, and placed polished side down in clean polystyrene cells. The cleaned and dried substrates were stored inside the humidified glove box overnight ( $>8$ hours) to equilibrate before the contacting experiments.

Wooden dowels were glued to the unpolished side of each sample (General Purpose Hot-Melt glue, McMaster-Carr), and act as handles to hold the samples. Our tests showed that the wooden dowels and glue do not affect the charge measurements. After an experimental trial, the dowels were removed, and the samples were cleaned as described above and reused.

The rubbing of the samples was carried out as follows. The samples were held by the wooden dowels, one in each hand. The samples were brought together, so that the polished sides of the two samples were in planar contact. One sample was held still, and the other sample was translated in the pattern of a circle of radius $\approx 3 \mathrm{~mm}$ while maintaining planar contact. This motion continued for 30 seconds. After this contact, each sample was sequentially placed inside a Faraday cup located inside the glove box, where the Faraday cup was connected to an 
electrometer (Keithley 6517A) located outside of the glove box. The charge was directly measured and recorded by a Labview program. The background drift in our Faraday pail setup was $10^{-15}$ to $10^{-17} \mathrm{C} / \mathrm{s}$, depending on the humidity inside the glove box (higher drift with higher humidity), and the initial baseline charge was on the order of $10^{-12} \mathrm{C}$. Charge measurements above this threshold value were thus considered to be real. For every charging experiment, the initial charge on each sample was first obtained and then the charge after contacting was measured. The surface charge density was calculated based on the sample surface area $1 \mathrm{~cm}^{2}$.

X-ray photoelectron spectroscopy (XPS) was performed with a PHI VersaProbe XPS Microprobe. Spectra were collected from approximately three different spots on three different identically prepared quartz and sapphire samples, either immediately after cleaning or after the contact experiment; the results from these different samples were averaged.

\section{Results}

We first describe our modeling results. In Figure 2, we show the total energy of the system as a function of the separation distance between the two surfaces. Beyond a separation of $4 \AA$, the surfaces do not interact, due in part to the localized nature of the atomic orbital basis set. As the surfaces move closer to less than $4 \AA$, an attractive force develops between the surfaces, which manifests in Figure 2 as the decrease in energy as the surfaces come closer. At a separation of $2.4 \AA$, the attraction reaches a maximum, and the energy begins to increase as the separation between the surfaces decreases. The minimum energy separation between the surfaces is therefore approximately $2.4 \AA$; this separation would correspond to the equilibrium separation, and smaller separations would only be possible if an external force were applied to push the surfaces closer together. 
As the quartz and sapphire surfaces approach one another, the electron states from the two surfaces interact, and the electrons can redistribute between the quartz and sapphire slabs; i.e., electron transfer occurs. We use Mulliken population analysis to assess how the electrons partition to each slab. With Mulliken population analysis, the overlapping electron density between two atomic orbitals is split equally to the two atoms. By summing up electrons in atoms in the quartz slab and atoms in the sapphire slab, we can determine the charge on each slab. This analysis was carried out as a function of separation between the slabs, and the results are shown in Figure 3. The simulations show that quartz charges negatively and sapphire charges positively, in agreement with the experimental results. We address the magnitude of the charge in the Discussion section, but here we note that the charge on the surfaces while in contact does not correspond to the charge on the surfaces that remains after separation.

We now present our experimental results. Figure 4 shows cumulative measurements from a total of 91 trials of contact charging carried out between sapphire (0001) and quartz (0001) surfaces at various humidities. We believe these results correspond to saturated charge, in that further contact would not increase the charge. In all experimental trials, independent of humidity, sapphire was found to charge positively and quartz was found to charge negatively. The magnitude of the surface charge density increased with decreasing humidity, which is consistent with the usual influence of humidity on charging. ${ }^{15}$ The mean values for the charge densities as a function of humidity, with the corresponding standard errors (one standard deviation divided by the square root of the number of samples) are summarized in Table 1 . The mean charges of sapphire and quartz are measured independently, and are found to be the same in magnitude (but opposite in sign) at each value of humidity, which is evidence of the accuracy 
of the measurements. At the lowest humidity tested (5\% relative humidity) the magnitude of charge on the surfaces is approximately $10 \mu \mathrm{C} / \mathrm{m}^{2}$.

To assess the extent of material transfer during contact, we carry out XPS measurements. Figure 5a shows averaged XPS survey spectra collected from sapphire surfaces before (black) and after (red) contact with quartz. Before contact, the cleaned surface shows peaks corresponding to $\mathrm{Al}$ and $\mathrm{O}$ with a ratio of $~ 30: 52$, which is in agreement with the equilibrium surface composition. Peaks from $\mathrm{C}$ and $\mathrm{Si}$ are believed to have originated from contamination, either on the surface that could not be removed by the cleaning procedure or from room air during transfer of the samples into the instrument. Importantly, no significant differences were observed in the XPS-measured surface compositions before and after contact. Figure 5b shows the analogous averaged XPS survey spectra collected from quartz surfaces before (black) and after (red) contact with sapphire. Before contact, the cleaned surface shows peaks corresponding to $\mathrm{Si}$ and $\mathrm{O}$ with a ratio of $\sim 27: 58$, which is in agreement with the equilibrium surface composition. Again, peaks from $\mathrm{C}$ and $\mathrm{Al}$ are detected which we attribute to contamination. After contact, there is no significant change in the measured surface compositions. Together, these results indicate that the amount of material transfer upon contact was insignificant, and we can conclude that material transfer did not play a significant role in the charge transfer mechanism.

We looked for a simple property to correlate the direction of charge transfer, and carried out further calculations and experiments in this regard. One possible property is the electrostatic potential energy, which represents the amount of work to move a positive charge to a specific position. The results for the electrostatic potential energy are shown in Figure 6. Since sapphire has lower electrostatic potential energy than quartz, it is more likely to attract a positive charge. 
This result is in agreement with the charging polarity observed experimentally. To test the generality of this correlation between the electrostatic potential energy and the direction of charge transfer, we carried out brief studies with periclase (MgO crystal with the rocksalt structure). Since the periclase crystal structure is cubic, we could not perform calculations of periclase in contact quartz or sapphire (due to the different symmetry of the periodic boundary conditions). However, we could obtain the average electrostatic potential energy relative to vacuum. We find that the order of the values of the average electrostatic energy relative to vacuum is periclase $>$ sapphire $>$ quartz; thus based on the electrostatic potential energy, we would predict periclase to develop a positive charge when contacted with either sapphire or quartz. To assess this prediction, contact charging experiments involving periclase were carried out at a relative humidity of $1 \%$. For contact between sapphire and periclase we measured average charges of $13 \pm 3.0$ and $-7.6 \pm 2.1 \mu \mathrm{C} / \mathrm{m}^{2}$, respectively; for contact between periclase and quartz, we measured average charges of $14 \pm 2.4 \mu \mathrm{C} / \mathrm{m}^{2}$ and $-10 \pm 2.8 \mu \mathrm{C} / \mathrm{m}^{2}$, respectively. These experimental results indicate a triboelectric series ordered from most positve to most negative as sapphire $>$ periclase $>$ quartz, which contradicts the ordering of the electrostatic potential energy. Thus the direction of charging in contact electrification cannot be simply predicted by the electrostatic potential energy.

\section{Discussion and Conclusions}

Contact charging is a non-equilibrium process that occurs as follows. When the surfaces of two materials are far away from each other, the equilibrium state of the system corresponds to neutral surfaces. As the material surfaces come very close (within a few Angstroms) or into contact, the equilibrium state can change such that the two surfaces are no longer neutral (due to 
electron transfer between the surfaces). As the surfaces move away from one another, the gap between the surfaces creates an energy barrier that inhibits the transfer of charge back to the neutral equilibrium state; the height of this barrier increases as the surfaces move apart. Once the surfaces move more than a few Angstroms apart, the barrier is so large that "electron backflow" is effectively zero; Lowell and Rose-Innes estimated this distance to be approximately $10 \AA .{ }^{16}$ In this way, some of the charge that had transferred when the surfaces were in contact becomes "stuck", and this "stuck charge" is what we would observe in contact charging experiments. The "stuck charge" is a non-equilibrium situation, which is observed because the slow kinetics of the electron backflow prevents the system from reaching the equilibrium state with neutral surfaces.

Our calculations are based on the equilibrium (lowest energy) state of the system. Thus in the calculations charge is always able to flow back to the other surface during separation (in contrast to actual contact charging experiments). The process in our calculations is fully reversible and as the surfaces come together, the charge on the surfaces follows the curves in Figure 3; likewise, as the surfaces move apart, the charge on the surfaces again follows the curves in Figure 3, but in reverse so that the charge on the surfaces goes to zero as the surfaces move far apart. Our methodology cannot determine the point at which charge gets "stuck" due to the energy barrier created by separation. Unfortunately, a non-equilibrium method that addresses the quantum dynamics of the electrons as the surfaces move apart is far too computationally intensive to be feasible.

However, we can test the applicability of our calculations to contact charging as follows. Figure 7 shows calculation results for the charge on a sapphire surface contacted with quartz on a log scale (the charge on the corresponding quartz surface is equal in magnitude, but opposite in 
sign). Results at separations greater than $3.2 \AA$ are dominated by noise (i.e., numerical precision in the calculation) because the charge values become very small. Therefore, we use an exponential fit to extrapolate our data to larger separations; an exponential functional dependence is expected because the charge transfer is due to the overlap of electron wavefunctions, and wavefunctions decay exponentially with distance. ${ }^{17}$ We can assume that the charge on the surfaces will follow the values given by this extrapolation until a point is reached where electron backflow can no longer occur. We see that this extrapolation reaches the value of the charge found experimentally $\left(10 \mu \mathrm{C} / \mathrm{m}^{2}\right)$ at about $8 \AA$; so if electron backflow ceases to occur for separations greater than $8 \AA$, the charge of $10 \mu \mathrm{C} / \mathrm{m}^{2}$ would be "stuck" on the surfaces as the surfaces move far apart. Thus our calculation results, combined with a physically a reasonable distance for where the electron backflow would cease to occur, ${ }^{16}$ leads to the charge density 10 $\mu \mathrm{C} / \mathrm{m}^{2}$ that is found in our experiments. Thus, we conclude that our first-principles calculations are consistent the experimental results.

While our first-principles calculations are consistent the experimental results, we note a couple of important caveats: (a) the distance taken for where electron backflow ceases (8 $\AA$ ), while being physically appropriate, is nonetheless arbitrary; other physically appropriate distances for this parameter would give results that differ somewhat from experiment. (b) the extrapolation of charge density data less than $32 \AA$ to estimate the value of the charge density at $8 \AA$ will very likely have inaccuracies. For these reasons we note that our calculation results cannot be considered to be predictive or definitively in agreement with experiment; rather, our analysis shows that the calculation results are consistent with experiment.

The charge transfer results of our first-principles calculations, which address only an electron transfer mechanism of charge transfer, can describe the charge transfer results of our 
experiments. In this way our results suggest that it is an electron transfer mechanism that underlies charge transfer in the quartz-sapphire system. We note that ion transfer and material transfer mechanisms have been shown to play roles in at least some circumstances. The results of our XPS experiments do not show evidence of material transfer, and thus we conclude that the material transfer mechanism is not important for the quartz-sapphire system, perhaps because these materials are both very hard. We cannot rule out an ion transfer mechanism, and of course the possibility exists that the consistency of the calculations with experiment is fortuitous and that an ion transfer mechanism not addressed in our calculations actually dominates. Finally we note that it is likely that the different possible charge transfer mechanisms (electron, ion, material) dominate under different conditions and for different materials, and if indeed it an electron transfer mechanism dominates for the quartz-sapphire system at low humidity this will not necessarily generalize to other situations and materials.

\section{AUTHOR INFORMATION}

E-mail: daniel.lacks@case.edu and mohan@case.edu

\section{Notes}

The authors declare no competing financial interests.

\section{ACKNOWLEDGMENTS}


This material is based upon work supported by the National Science Foundation under grant numbers CBET-1235908 and DMR-120648. The calculations were carried out using the computational resources of the Ohio Supercomputing Center. 
Table 1. Mean charge densities in contact charging experiments between sapphire and quartz. The error estimate is the standard error.

\begin{tabular}{lll}
\hline $\begin{array}{l}\text { Relative Humidity } \\
(\%)\end{array}$ & $\begin{array}{l}\text { Sapphire charge density } \\
\left(\mu \mathrm{C} / \mathrm{m}^{2}\right)\end{array}$ & $\begin{array}{l}\text { Quartz charge density } \\
\left(\mu \mathrm{C} / \mathrm{m}^{2}\right)\end{array}$ \\
\hline \hline 5 & $10.01 \pm 1.06$ & $-8.66 \pm 0.98$ \\
20 & $7.50 \pm 1.00$ & $-6.95 \pm 0.98$ \\
70 & $6.07 \pm 0.76$ & $-6.13 \pm 0.70$ \\
95 & $0.79 \pm 0.18$ & $-1.13 \pm 0.27$ \\
\hline
\end{tabular}




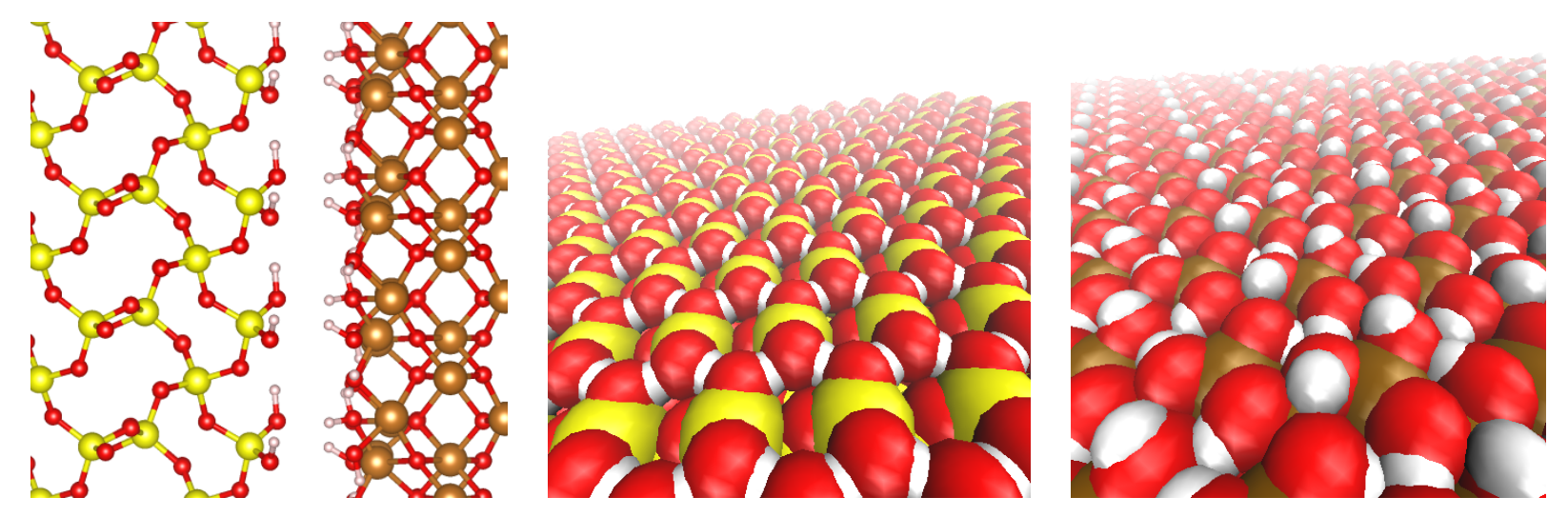

Figure 1. System examined in the calculations. (a) view from the side, with quartz on left and sapphire on right; (b) quartz [0001] surface; (c) sapphire [0001] surface. In these figures oxygen is red, silicon is yellow, aluminum is brown, and hydrogen is white. 


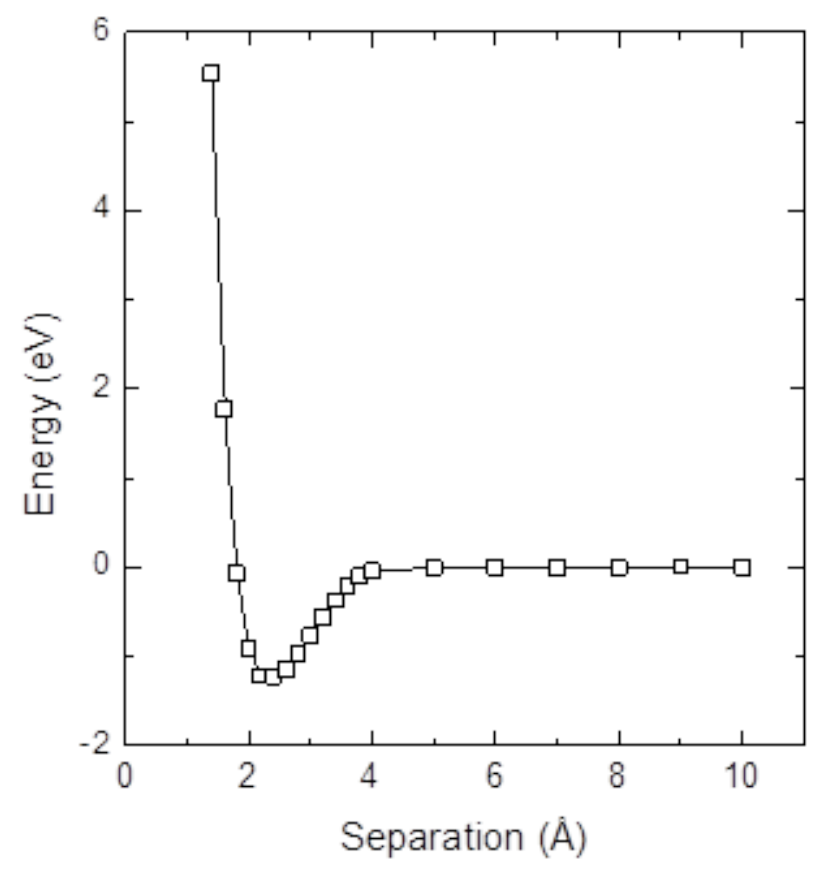

Figure 2. Calculation results for the total energy of the quartz-sapphire system as a function of separation between the quartz and sapphire [0001] surfaces. The energy given is relative to the energy in the limit of large separation. 


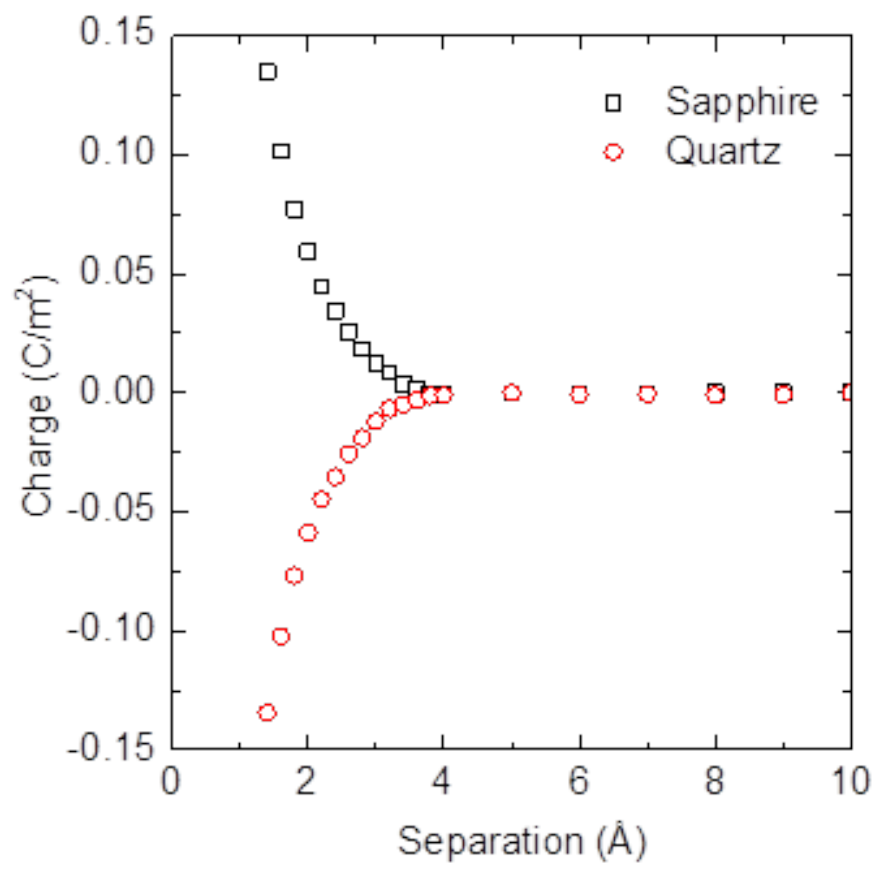

Figure 3. Charge on sapphire and quartz slabs obtained from calculations, using Mullikan Population Analysis. 


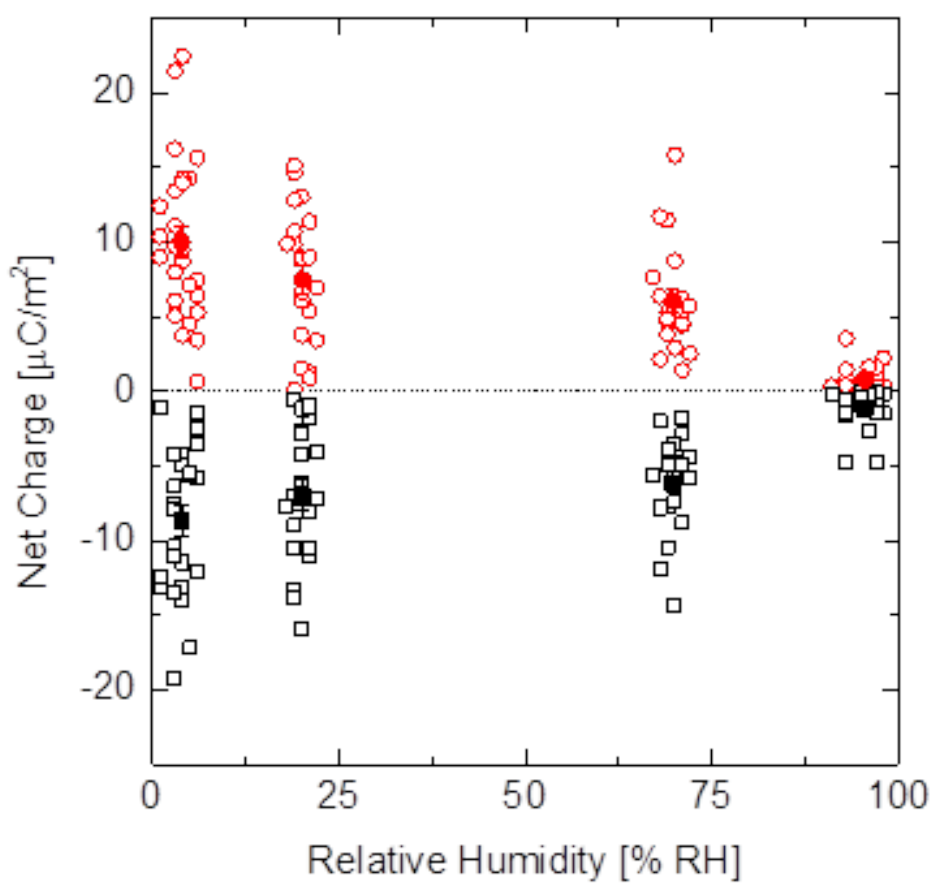

Figure 4. Net surface charge densities from contact charging of sapphire against quartz; open circles (○) represent sapphire trials, open squares $(\square)$ represent quartz trials, filled circles $(\bullet)$ represent sapphire average values, filled squares($(\boldsymbol{\bullet})$ represent quartz average values. 

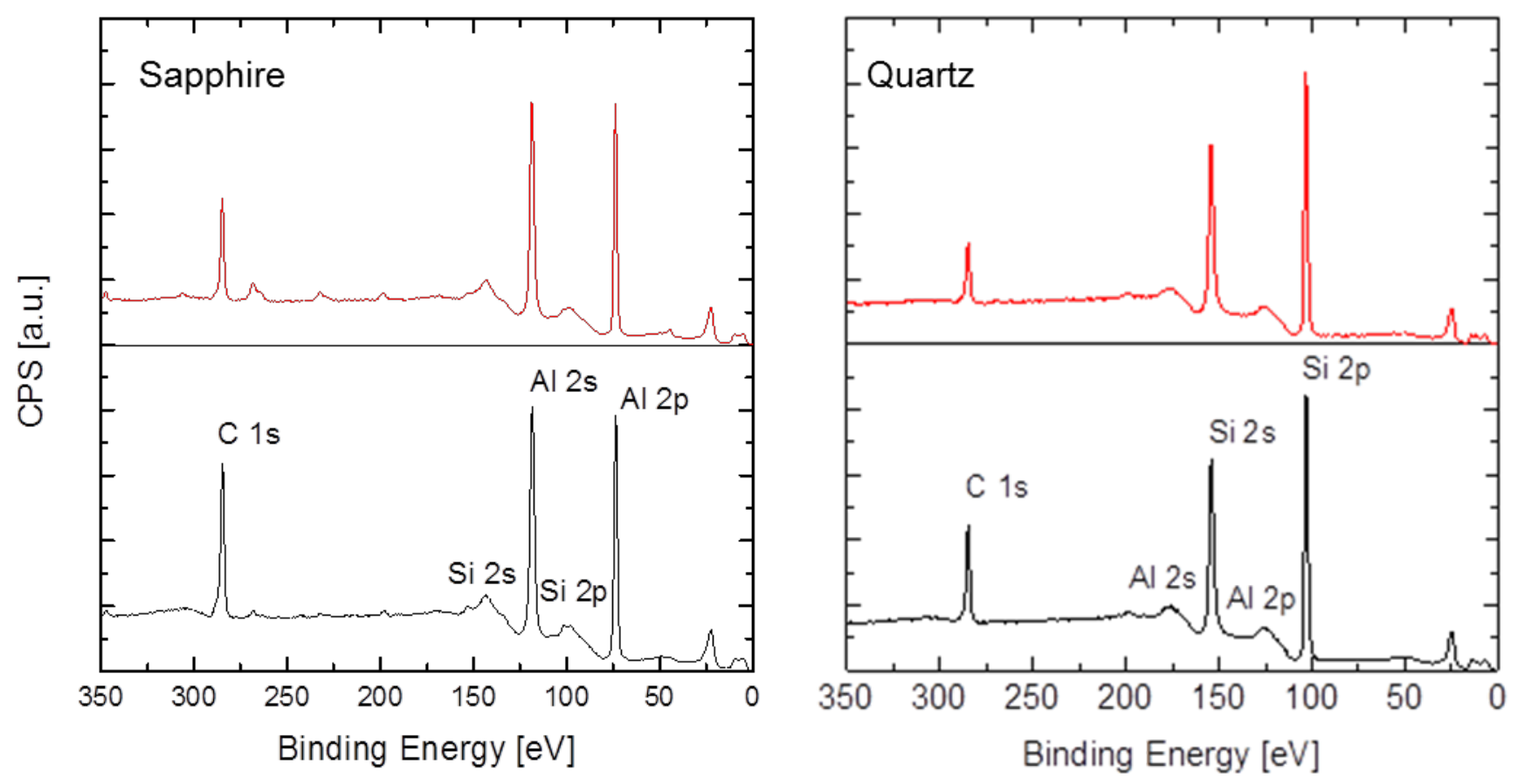

Figure 5. Averaged XPS spectra collected from freshly cleaned surfaces (black), and surfaces after rubbing as described in text (red). (a) [0001] sapphire; (b) [0001] quartz. 


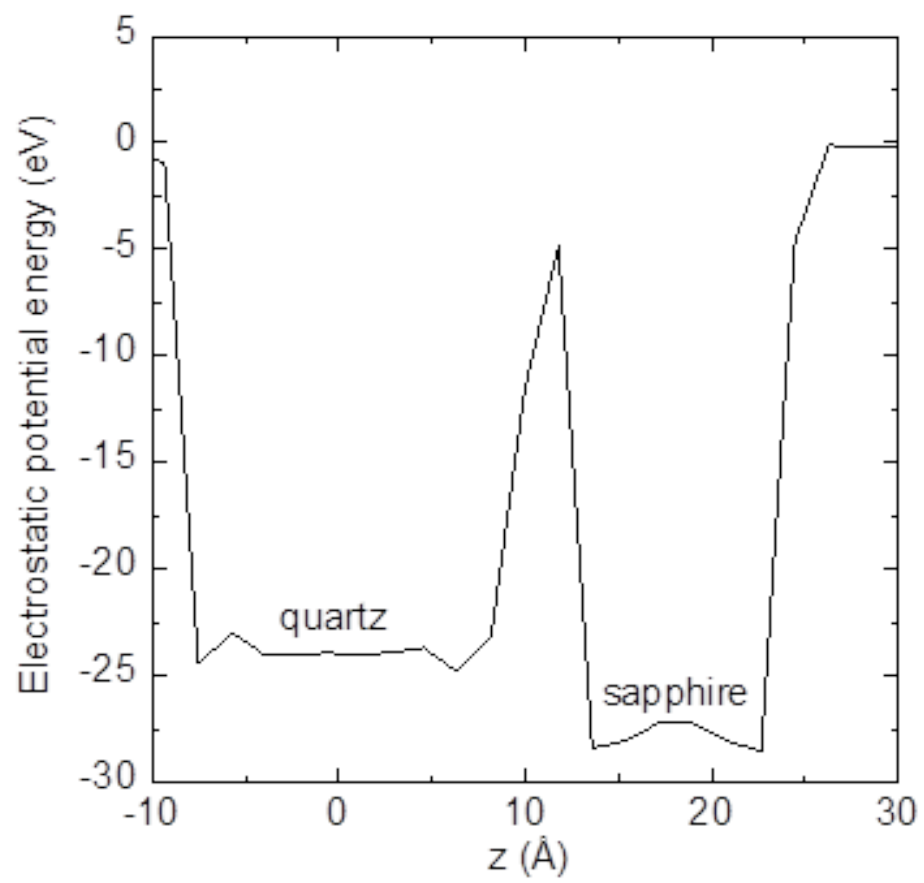

Figure 6. Electrostatic potential energy obtained from DFT calculations of surfaces separated by 3 Angstrom. 


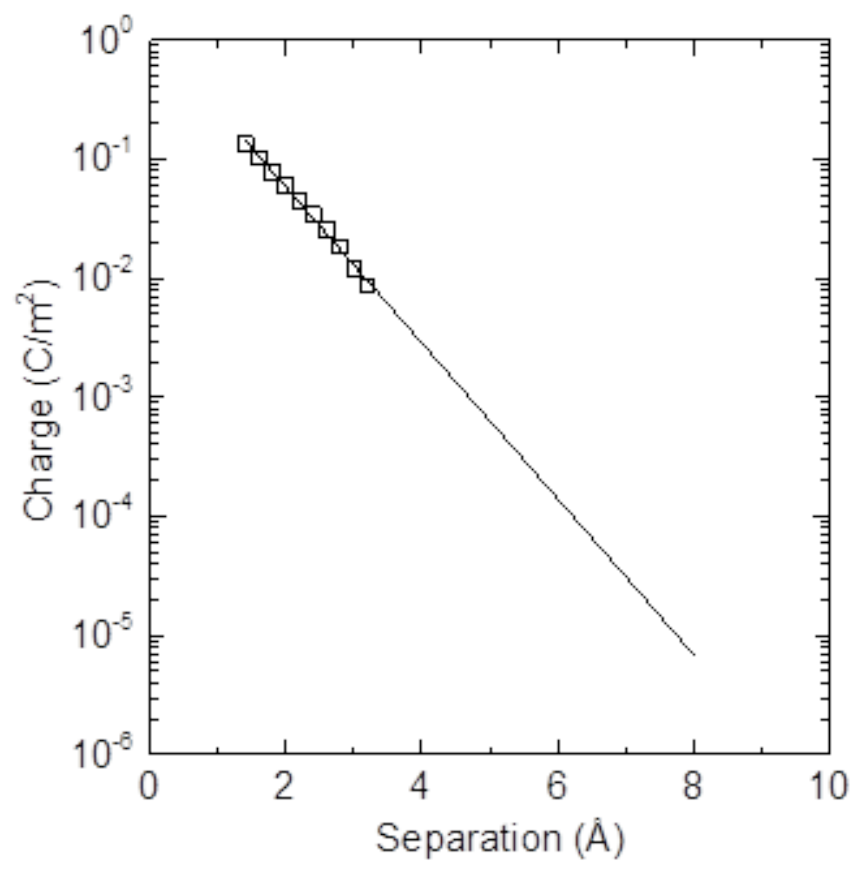

Figure 7. Charge on sapphire slab obtained from calculations. The line is an exponential fit to the data. 


\section{REFERENCES}

1 Lacks, D. J.; Sankaran, R. M. Contact Electrification of Insulating Materials J. Phys. D: Appl. Phys. 2011, 44, 453001, DOI: 10.1088/0022-3727/44/45/453001.

2 McCarty, L. S.; Whitesides, G. M. Electrostatic Charging Due to Separation of Ions at Interfaces: Contact Electrification of Ionic Electrets. Angew. Chem., Int. Ed. 2008, 47, 21882207, DOI: 10.1002/anie.20070181.

3 Liu, C. Y.; Bard, A. J. Electrostatic Electrochemistry at Insulators. Nature Materials 2008, 7, 505-509, DOI: 10.1038/nmat2160.

4 Lacks, D. J. The Unpredictability of Electrostatic Charging. Angew. Chem., Int. Ed. 2012, 51, 6822, DOI: 10.1002/anie.201202896.

5 Baytekin, H. T.; Patashisnki, A. Z.; Branicki, M.; Baytekin, B.; Soh, S. Grzybowski, B. A. The Mosaic of Surface Charge in Contact Electrification. Science 2011, 333, 308-312 DOI: 10.1126/science.1201512.

6 Baytekin, H. T.; Baytekin, B.; Incorvati, J. T.; Grzybowski, B. A. Material Transfer and Polarity Reversal in Contact Charging. Angew. Chem., Int. Ed. 2012, 51, 4843-4847, DOI: 10.1002/anie.201200057.

15 Perdew, J. P.; Burke, K.; Ernzerhof, M. Generalized Gradient Approximation Made Simple. Phys. Rev. Lett. 1996, 77, 3865-1396, DOI: 10.1103/PhysRevLett.78.1396.

8 Soler, J. M.; Artacho, E.; Gale, J. D.; García, A.; Junquera, J.; Ordejón, P.; Sánchez-Portal, D. The SIESTA Method for ab initio order-N Materials Simulation. J. Phys.: Condens. Matter 2002, 14, 2745-2779, DOI: 10.1088/0953-8984/14/11/302. 
9 Levien, L.; Prewitt, C. T.; Weidner, D. J. Structure and Elastic Properties of Quartz at Pressure. Am. Mineral. 1980, 65, 920-930, DOI: 0003-004x/80/0910-092

10 Lewis, J.; Schwarzenbach, D.; Flack, H. D. Electric Field Gradients and Charge Density in Corundum, $\alpha$-Al2O3. Acta Crystallogr., Sect. A: Crystal Physics, Diffraction, Theoretical and General Crystallography 1982, 38, 733-739, DOI: 10.1107/S0567739482001478.

11 Yang, J.; Wang, E. G. Water Adsorption on Hydroxylated $\alpha$-Quartz (0001) Surfaces: From Monomer to Flat Bilayer, Phys. Rev. B 2006, 73, 035406, DOI: 10.1103/PhysRevB.73.035406.

12 Hass, K. C.; Schneider, W. F.; Curioni, A.; Andreoni, W. The Chemistry of Water on Alumina Surfaces: Reaction Dynamics from First Principles, Science 1998, 282, 265-268, DOI 10.1126/science.282.5387.265.

13 Zhang, D.; Wang, Y.; Gan, Y. Characterization of Critically Cleaned Sapphire SingleCrystal Substrates by Atomic Force Microscopy, XPS and Contact Angle Measurements. Appl. Surf. Sci. 2013, 274, 405-417, DOI:10.1016/j.apsusc.2012.12.143

14 Henke, L.; Nagy, N.; Krull, U. J. An AFM Determination of the Effects on Surface Roughness Caused by Cleaning of Fused Silica and Glass Substrates in the Process of Optical Biosensor Preparation. Biosens. Bioelectron. 2002, 17 (6-7), 547-555, DOI: 10.1016/S09565663(02)00012-X

15 V. Albrecht, A. Janke, E. Németh, S. Spange, G. Schubert, F. Simon. Some Aspects of the Polymers' Electrostatic Charging Effects”, J. Electrostat. 2009, 67(1), 7-11, DOI: 10.1016/j.elstat.2008.10.002. 
16 Lowell J.; Rose-Innes, A. C. Contact Electrification. Adv. Phys. 1980, 29, 947-1023.

17 Karplus, M.; R. Porter, N. Atoms and Molecules, W. A. Benjamin 1970. 